American Journal of Immunology 4 (1): 1-7, 2008

ISSN 1553-619X

(C) 2008 Science Publications

\title{
An Analysis of the Creatine Kinase Isoenzyme and Cardiac Troponin T Levels in a Polymyositis Patient
}

\author{
${ }^{1}$ R.M. Golding, ${ }^{2}$ L.G.F. Giles and ${ }^{3}$ E.M. Sokoya \\ ${ }^{1}$ The University of New South Wales, Sydney, New South Wales 2052, Australia \\ ${ }^{2}$ Murdoch University, South Street, Murdoch, Western Australia 6150, \\ Australia and James Cook University of North Queensland, Townsville, Queensland 4810, Australia \\ ${ }^{3}$ Department of Anesthesiology, Baylor College of Medicine, Houston, TX 77030, USA
}

\begin{abstract}
From an extensive model developed to interpret physiological processes we are able to show that the observed elevated creatine kinase-myoglobin (CK-MB) and serum concentration of cardiac troponin $\mathrm{T}(\mathrm{cTnT})$ values for a particular patient are associated with the inflammatory disease, polymyositis, and not myocardial injury. This highlights that caution must be exercised in using elevated CK-MB and cTnT levels as evidence for myocardial damage in patients with chronic muscle degeneration such as polymyositis.
\end{abstract}

Key words: Polymyositis, prednisolone, blood pressure, heart rate, erythrocyte sedimentation rate, creatine kinase, creatine kinase isoenzyme, cardiac troponin $\mathrm{T}$

\section{INTRODUCTION}

Inflammatory muscle diseases, such as polymyositis, result in a number of physiological changes such as increased levels of erythrocyte sedimentation rate (ESR) and serum creatine kinase (CK). When an inflammatory process is present, the high proportion of fibrinogen in the blood causes red blood cells to adhere to one another, thereby increasing the ESR. The ESR is a non-specific measure of inflammation. CK is a cytoplasmic muscle enzyme responsible for the regeneration of ATP. The process of muscle degeneration causes muscle cells to lyse, releasing their contents into the bloodstream. Since most of the CK in the body normally exists in muscle, a rise in the amount of $\mathrm{CK}$ in the blood indicates that muscle damage is present. We have previously shown that serum CK is an excellent index for assessing the optimal dosage of the corticosteroid, prednisolone, to decrease muscle inflammation due to the autoimmune disease polymyositis ${ }^{[1,2]}$. CK exists as three different isoenzymes: CK-MM (skeletal muscle), CK-MB (myocardium) and CK-BB (brain). CK-MB is expressed both in myocardial and striated muscle and has been used as a marker of both cardiac disease ${ }^{[3]}$ and inflammatory diseases ${ }^{[4]}$.

Troponins are a group of proteins that regulate the calcium mediated interaction of actin and myosin. Troponin $\mathrm{T}$ is a subunit of troponin that binds to tropomyosin thereby forming a troponin-tropomyosin complex within muscle tissue. Serum concentrations of cardiac troponin $\mathrm{T}$ values above $0.10 \mathrm{ng} \mathrm{mL}^{-1}$ are regarded as a highly sensitive marker of myocardial injury ${ }^{[5]}$. Some studies have reported that cTnT can be used to discriminate between myocardial disease and skeletal muscle disease ${ }^{[6]}$. However, other conditions such as polymyositis/dermatomyositis ${ }^{[7,8]}$, inclusion body myositis ${ }^{[9,10]}$, trauma ${ }^{[11]}$ and rhabdomyolysis ${ }^{[12]}$ have been reported to also result in increased levels of cTnT.

Over some fourteen years we have monitored and managed a patient with an inflammatory muscle disease $^{[1,2,13]}$ who has consulted with various medical specialists over the years. Furthermore, the patient does not have a myocardial problem as judged by the lack of symptoms and normal heart physiology rather than by ECG and echocardiogram. Therefore, the CK-levels have been equated to normal heart physiology. It should be noted that CK-MB and cTnT are not specific markers for cardiac muscle and that these markers are usually employed as biochemical markers in cases of acute ischaemic damage. We have suggested that in this particular patient, the muscle inflammatory initiation involved amlodipine besylate (Norvasc) ${ }^{[14]}$. A muscle biopsy, two years after the initiation of the muscle disease by amlodipine besylate, revealed severe muscle inflammation due to polymyositis ${ }^{[14]}$. Prednisolone was used therapeutically by the patient to minimize the muscle attack. The optimum dose for this particular patient was determined to be $62.5 \mathrm{mg}$ every second 
day ${ }^{[15]}$. This dose was chosen in order to maximize the reduction of the chemical muscle attack whilst significantly reducing the muscle inflammation and minimizing the drug concentration to ensure nominal drug side effects. Eight years later a second muscle biopsy revealed no muscle inflammation ${ }^{[2]}$ but ragged red fibres were observed that were attributed to mitochondrial changes that occur in the later stages of inclusion body myositis ${ }^{[16]}$. Nonetheless, while we acknowledge that polymyositis can take different courses, no rimmed vacuoles were found in the two muscle biopsies to suggest the presence of inclusion body myositis. Furthermore, CK levels are thought not to always correspond to the degree of muscle damage. Bearing in mind the above, we have been successful in creating a model that enables us to monitor and control the inflammatory disease using serum CK as an index of disease progression and prednisolone as a therapeutic strategy. From the model, we have developed a method for extracting muscle information from serum creatine kinase measurements $^{[2,15]}$.

Since long-term steroid use may result in the development of myocardial diseases ${ }^{[17]}$, in this reserch we examined the serum levels of CK-MB and cTnT in the same polymyositis patient who used a standardized prednisolone dose without the concomitant use of a further immunosuppressive drug, hence eliminating possible additional adverse side effects.

\section{MATERIALS AND METHODS}

After many years of analysis, we were able to determine the appropriate drug regimen to minimize muscle damage in a particular polymyositis patient. This regimen was adopted on July 27, 2001 that included $62.5 \mathrm{mg}$ prednisolone every second day, $2 \mathrm{mg}$ of odrik (trandolapril) and $450 \mathrm{mg}$ Ambrotose (a natural product by Mannatech) daily. Systolic pressure, diastolic pressure and heart rate were measured on a daily basis. Prednisolone was administered in the morning of day one and blood pressure and heart rate was recorded $12 \mathrm{~h}$ later and then $36 \mathrm{~h}$ later on day 2 .

Erythrocyte sedimentation rate (ESR), serum creatine kinase $(\mathrm{CK})$, creatine kinase isoenzyme myocardium (CK-MB) and cardiac troponin T (cTnT) were recorded every six weeks by the Queensland Medical Laboratories. Serum CK was measured using a COBAS Integra 800, whereas the CK-MB and cTnT were measured with an Elecsys 2010 (Roche) or a COBAS 6000 Analyser. Mannatech natural products (Mannatech Incorporated, Coppell, Texas, USA) taken during this study approximated $450 \mathrm{mg}$ of Ambrotose day $^{-1}$, three Plus tablets and one PhytAloe tablet day ${ }^{-1}$.
Note that in a previous study ${ }^{[1]}$ it was shown that a reaction occurs between the prednisolone and the Mannatech. This resulted in the need to increase the prednisolone from $50 \mathrm{mg}$ every second day to $62.5 \mathrm{mg}$ to maintain the appropriate prednisolone attack on the chemical or chemicals responsible for the muscle disease.

Data analysis: To analyse the data we may group the results from July 27, 2001 to December 3, 2007 as follows:

Period (i): July 27, 2001 to December 3, 2007-ESR and CK measurements were recorded every six weeks.

Period (ii): August 8, 2001 to December 3, 2007-CKMB measurements were recorded every six weeks.

Period (iii): March 28, 2007 to December 3, 2007cTnT measurements were recorded every six weeks.

As background it is important to understand how we have used specific measurements to interpret the processes taking place within the patient in a meaningful way. We shall outline the background as follows:

- $\quad$ Prior to 4 Feb 1994 the patient had no background of any disease and took no drugs. Furthermore, the averages for the physiological measurements of systolic, diastolic, pulse pressure and heart rate were $142 \mathrm{mmHg}, 89 \mathrm{mmHg}, 53 \mathrm{mmHg}$ and $71 \mathrm{~min}^{-1}$ respectively

- $\quad$ On 4 Feb 1994 amlodipine besylate (norvasc) was taken. Over the next two years the systolic, diagnostic, pulse pressure and heart rate average were $132 \mathrm{mmHg}, 83 \mathrm{mmHg}, 50 \mathrm{mmHg}$ and $79 \mathrm{~min}^{-1}$ respectively. Comparing the results prior to and after the introduction of norvasc show clearly that the systolic and diastolic values were significantly reduced but the heart rate was statistically significantly increased. See Golding, Giles and Sokoya ${ }^{[14]}$ for the details. Replacing norvasc by trandolapril (odrik) during 1995 made no change

- On 22 Apr 1995 the first serum creatine kinase (CK) measurement was made and it was 949 Ul /I

- In late Feb 1996 a muscle biopsy showed that there was severe muscle inflammation due to chronic active polymyositis. The inflammatory component with muscle necrosis and regeneration was set in a background of disordered muscle fibre architect 
where a significant number of muscle fibres had been lost and replaced by fat and fibrous tissue. Many of the surviving fibres, apart from those that showed active necrosis, were quite artrophic. These chronic myopathic changes correlate quite strongly with reduced muscle power

- From 11 Apr 1996, when 60 mg of prednisolone day $^{-1}$ was taken for six days and then reduced to $50 \mathrm{mg} \mathrm{day}^{-1}$, the $\mathrm{CK}$ level steadily reduced to $160 \mathrm{Ul} / 1$ by 3 May 1996. The prednisolone then was gradually reduced to $20 \mathrm{mg} \mathrm{day}^{-1}$ when the CK stabilised around 130 Ul/1 by 2 Oct 1996

- From May 1996 until July 2001 variations in the prednisolone (50-20 mg day ${ }^{-1}$ and 50-75 mg every second day) and odrik ( 2 and $3 \mathrm{mg} \mathrm{day}^{-1}$ ) and the introduction of the natural product Ambrotose gave a wide range of $\mathrm{CK}$-values and blood pressure values. In an attempt to understand the processes taking place in a way that we may use the data obtained in a meaningful way, a fundamental scientific model was developed. See Golding ${ }^{[15]}$. This may be expressed as follows:

Physiological measurements lead to the development of a scientific model to explore the physiological processes. This then may be used to estimate the physiological measurements from the model, in other words, the scientific model and physiological processes lead to an understanding of the physiological measurements. A powerful aspect of this approach is that it enables optimum conditions to be determined to combat the muscle disease and a method of monitoring the disease. Furthermore, it highlights the dangers of using multi-patient statistics in monitoring and controlling a specific disease such as muscle inflammation. The model shows that a longitudinal study is essential.

- The model for muscle inflammation may be summarised by a specific number of rate processes as follows where:

$\mathrm{M}=$ Muscle concentration

$\mathrm{CK}=$ Creatine kinase concentration

$\mathrm{P}=$ Anti-muscle antibodies concentration

$\mathrm{X}=$ Anti-muscle antibodies concentration

$\mathrm{S}=$ Prednisolone concentration

$\mathrm{NP}=$ Natural product (Ambrotose) concentration

The n-value is associated with the release of creatine kinase, the m-value is associated with the antimuscle antibody interaction with the steroid and the $\mathrm{m}_{\mathrm{x}}{ }^{-}$ value is associated with substance $\mathrm{X}$ interaction with steroid.

The Z-value does not need to be defined; it represents a wide range of chemicals associated with each specific reaction and its value has no effect on the model outcomes.

The k-values define the rate processes. The arrows used in defining the following rate processes may be expressed as leads to.

The first equation describes the normal muscle (M) and creatine kinase $(\mathrm{CK})$ reactions:

$$
\mathrm{M} \rightarrow \mathrm{CK}+\mathrm{M}+\mathrm{Z} \quad\left(\mathrm{k}_{1}\right)
$$

The natural decay of the creatine kinase is given as:

$$
\mathrm{CK} \rightarrow \mathrm{Z} \quad\left(\mathrm{k}_{2}\right)
$$

To describe the muscle anti-muscle antibody interaction we express the muscle attack as:

$$
\mathrm{M}+\mathrm{P} \rightarrow \mathrm{n} \mathrm{CK}+\mathrm{Z} \quad\left(\mathrm{k}_{3}\right) \text { polymyositis }
$$

The natural decay of $\mathrm{P}$ follows as:

$$
\mathrm{P} \rightarrow \mathrm{Z} \quad\left(\mathrm{k}_{4}\right)
$$

The steroid interaction with the muscle-attacking substance or substances, $\mathrm{P}$, as:

$$
\mathrm{P}+\mathrm{m} \mathrm{S} \rightarrow \mathrm{Z} \quad\left(\mathrm{k}_{5}\right)
$$

The natural steroid decay is described by:

$$
\mathrm{S} \rightarrow \mathrm{Z} \quad\left(\mathrm{k}_{6}\right)
$$

A natural product or additional drug interaction may be expressed as:

$$
\mathrm{NP}+\mathrm{S} \rightarrow \mathrm{Z} \quad\left(\mathrm{k}_{7}\right)
$$

To describe the muscle anti-muscle antibody interaction we express the muscle attack as:

$$
\mathrm{M}+\mathrm{X} \rightarrow \mathrm{Z} \quad\left(\mathrm{k}_{\mathrm{x} 3}\right) \text { inclusion body myositis }
$$

The natural decay of $\mathrm{X}$ follows as:

$$
\mathrm{X} \rightarrow \mathrm{Z} \quad\left(\mathrm{k}_{\mathrm{x} 4}\right)
$$

The steroid interaction with the muscle-attacking substance or substances, $\mathrm{X}$, as: 
Am. J. Immunol., 3 (1): 1-7, 2008

$$
\mathrm{X}+\mathrm{m}_{\mathrm{x}} \mathrm{S} \rightarrow \mathrm{Z}\left(\mathrm{k}_{\mathrm{x} 5}\right)
$$

Rp: Rate of production of the anti-muscle antibodies, $\mathrm{P}$ $\mathrm{Rx}$ : Rate of production of the anti-muscle antibodies, $\mathrm{X}$ Rs: Rate of intake of prednisolone

The model is independent of the specific chemical or chemicals representing $\mathrm{P}$ and $\mathrm{X}$.

The details of how to use these postulated interactions are given by Golding ${ }^{[15]}$. Its potential value in the monitoring and management of inflammatory muscle disease is detailed in Golding and Giles ${ }^{[2]}$. Golding and Golding ${ }^{[1]}$ illustrate controlling and monitoring polymyositis under steroid therapy. We may explore, for example, any observed changes in the heart rate from our model. We shall illustrate this by assuming that the physiological processes have not changed over the years. The model shows that the chemicals attacking the muscles affect the heart rate in a particular way and may be described as heart rate $=$ constant+0.18 S, Golding, Giles and Sokoya ${ }^{[14]}$. Prior to 4 Feb 1994, from background (1), the constant was $71 \mathrm{~min}^{-1}$ with $\mathrm{S}=0$. After $4 \mathrm{Feb} 1994$, from background (2), the constant is $79 \mathrm{~min}^{-1}$. If we use $62.5 \mathrm{mg}$ of prenisolone every second day then we have approximately $S=62.5 \mathrm{mg}$ for about half a day but after almost two days it would be greatly reduced to approximately zero. Hence, on day one the model suggests that the heart rate should be about $90 \mathrm{~min}^{-1}$ and on day two about $79 \mathrm{~min}^{-1}$ as observed. Importantly the heart rate-value and the way it changes have not varied for fourteen years. Furthermore, the evidence is that if the chemicals attacking the muscles were reduced to zero the heart rate would return to $71 \mathrm{~min}^{-1}$.

A similar model has been developed and used successfully in modelling of responses of cerebral blood vessels to changing intraluminal pressure, Golding and Golding ${ }^{[18]}$.

\section{RESULTS}

For period (i) the ESR data is shown in Fig.1. From Fig. 1, prior to 1000 days, there are three high values above $30 \mathrm{~mm} \mathrm{~h}^{-1}$ that may have occurred from an abnormal event such as an insect bite. The two high values on days 2152 and 2194 may be attributed to the consequence of two major falls of the patient that caused massive bruising. The mean ESR during the period (removing the six aberrant measurements above $30 \mathrm{~mm} \mathrm{~h}^{-1}$ ) was $18.23 \pm 3.88 \mathrm{~mm} \mathrm{~h}^{-1}$. This is comparable with the value given in Golding and Giles $^{[2]}$, namely, $18.98 \pm 6.61 \mathrm{~mm} \mathrm{~h}^{-1}$.

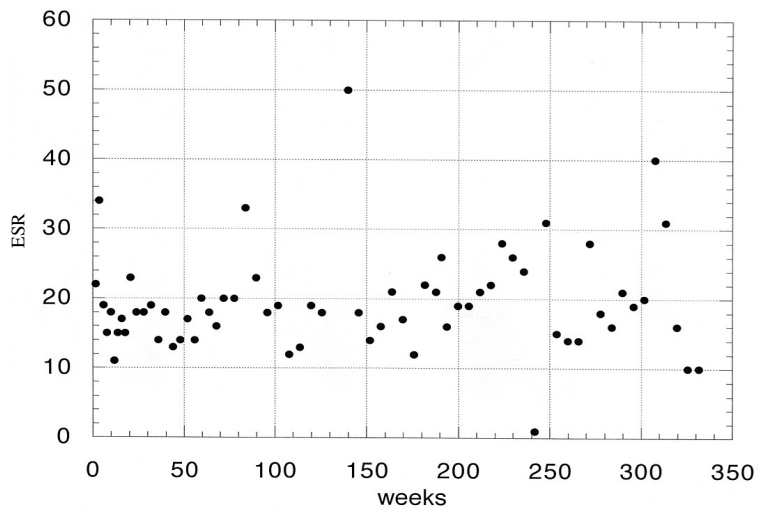

Fig. 1: The erythrocyte sedimentation rate in $\mathrm{mm} \mathrm{h}^{-1}$ measured every six weeks during the 6 year period in the patient with polymyositis

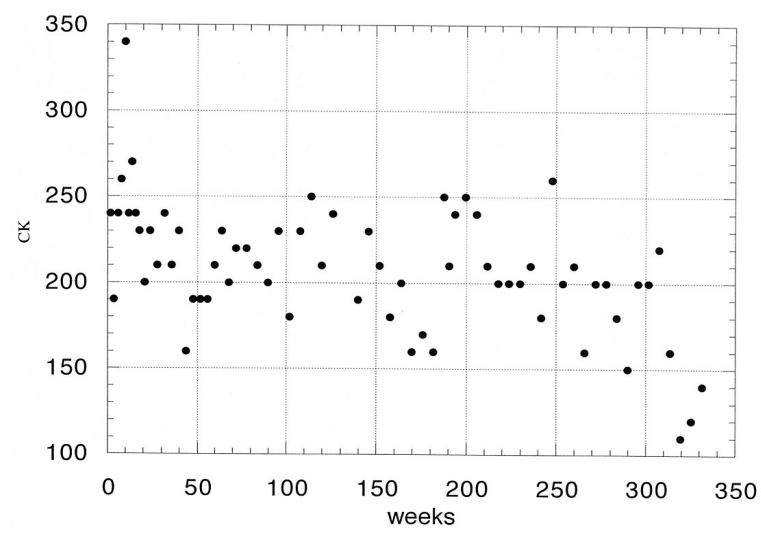

Fig. 2: The serum creatine kinase in IU/L for every six weeks during the 6 year period in the patient with polymyositis

For period (i) the serum $\mathrm{CK}$ results are shown in Fig. 2. The mean CK was $210.47 \pm 34.52$ IU/L. This value is comparable to $218.22 \pm 33.12 \mathrm{IU} / \mathrm{L}$, previously reported by Golding and Giles ${ }^{[2]}$. The last three values in Fig. 2 ranging from 110-140 IU/L are low due to the significant decrease in the patient's mobility as a result of the two major falls.

For period (ii) the mean CK-MB was 15.90 $\pm 2.38 \mathrm{IU} / \mathrm{L}$ and was time independent (Fig. 3). For period (iii) the mean cTnT was $0.14 \pm 0.02 \mathrm{IU} / \mathrm{L}$ and was time independent with a range from 0.11-0.17 IU/L.

For period (i) the patient's mean systolic pressure, over the period, was $144.81 \pm 10.50 \mathrm{mmHg}$, mean diastolic pressure was $79.45 \pm 6.57 \mathrm{mmHg}$ and the mean pulse pressure was $65.37 \pm 9.20 \mathrm{mmHg}$. These values mirror those given by Golding and Giles ${ }^{[2]}$. 
Am. J. Immunol., 3 (1): 1-7, 2008

Table 1: The yearly average values of the heart rate $12 \mathrm{~h}$ after taking prednisolone (Day one) and $36 \mathrm{~h}$ after taking prednisolone (Day two)

\begin{tabular}{llll}
\hline Year & $\begin{array}{l}\text { Day one } \\
\left(\mathrm{min}^{-1}\right)\end{array}$ & $\begin{array}{l}\text { Day two } \\
\left(\mathrm{min}^{-1}\right)\end{array}$ & $\begin{array}{l}\text { Difference } \\
\left(\mathrm{min}^{-1}\right)\end{array}$ \\
\hline 2001 & 93.035 & 80.034 & 13.00 \\
2002 & 91.474 & 77.123 & 14.35 \\
2003 & 93.095 & 80.545 & 12.55 \\
2004 & 91.949 & 78.918 & 13.03 \\
2005 & 94.836 & 81.986 & 12.85 \\
2006 & 91.926 & 81.127 & 10.80 \\
2007 & 91.755 & 77.228 & 14.53 \\
\hline
\end{tabular}

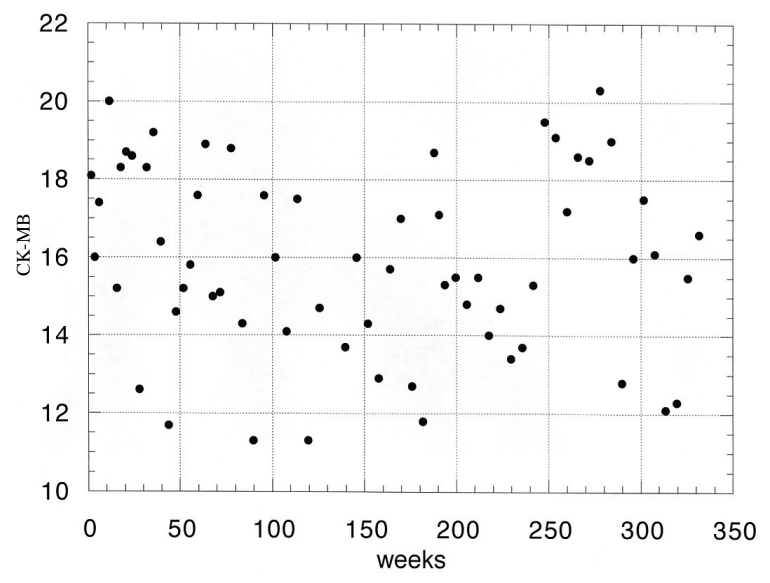

Fig. 3: CK-MB measurements recorded between August 8, 2001 and December 3, 2007 (Period ii)

Finally, we may examine the heart rate for day one (12 $\mathrm{h}$ following prednisolone intake) and day two (36 h following prednisolone intake). To do this we shall determine the average values for each year for the data given in period (i). The results are given in Table 1.

The heart rate results for day 1 and day 2 are plotted in Fig. 4. Although there are variations within the day 1 and day 2 values, the average difference is a constant of $13.02 \pm 1.24 \mathrm{~min}^{-1}$. This reinforces that we have no evidence of any changes taking place.

In summary, analysis of our three periods clearly show that from July 27, 2001 to December 3, 2007 we observed no change in the data over this period of almost seven years. This reinforces the earlier work that highlights that our patient has a muscle disease that can be attributed to chemicals attacking the muscles. It was suggested that the production of these chemicals was initiated by Norvasc ${ }^{[14]}$ taken on February 4, 1994. In addition to the elevated $\mathrm{CK}$ levels, the elevated CK-MB and cTnT levels are also a result of the inflammatory disease.

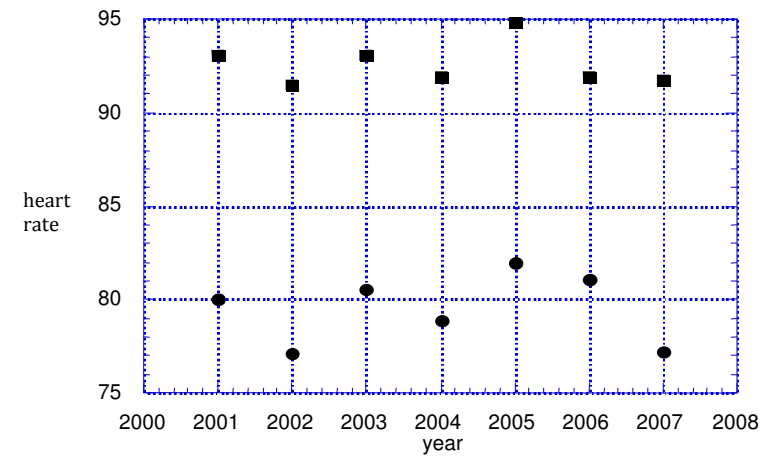

Fig. 4: The average heart rate for day 1 and day 2 for each year over the period given by Period (i)

\section{DISCUSSION}

As an overview of our results it should be noted that the serum CK-values are dependent on the normal muscle activity plus an increase due to the anti-muscle antibodies. To distinguish between the two is not precise and should be viewed with great care. However, the heart rates on day 1 and day 2 are directly related to the concentration of the anti-muscle antibodies. The average heart rate values for day 1 and day 2 are 92.3 and $79.6 \mathrm{~min}^{-1}$, respectively. If the rate of production of the anti-muscle antibodies tended to zero, the heart rates for day 1 and day 2 would tend to the value of $71 \mathrm{~min}^{-1}$ as observed prior to the initiation of the production of the anti-muscle antibodies ${ }^{[14]}$. Furthermore, we note that a reduction of the prednisolone concentration would be reflected in a decrease in the heart rate value difference ${ }^{[15]}$. All evidence indicates that no medical changes associated with this patient have occurred since the polymyostis initiation in early 1994. It should be noted that if the medication was stopped, the model shows that the CKlevel in the patient would reach $970 \mathrm{Ul} / \mathrm{l}$ in 200 days and that would have a major negative impact on the patient.

In Fig. 5 we illustrate how the model may be used to highlight the relationship between the steroid concentration versus the creatine kinase concentration. As a simple illustration using example (a), we shall choose the initial $\mathrm{CK}$-value as $983.33 \mathrm{Ul} / \mathrm{L}, \mathrm{P}$ as $250.0 \mathrm{Ul} / \mathrm{L}$, the steroid (S) as $62.5 \mathrm{mg}$ every second day with the natural product (Ambrotose) being taken daily. The calculated CK-value decreases with time to reach a constant value of $\mathrm{CK}=226.63(\mathrm{P}=26.56)$; example (b), we choose the initial $\mathrm{CK}$-value as $226.63, \mathrm{P}$ as 


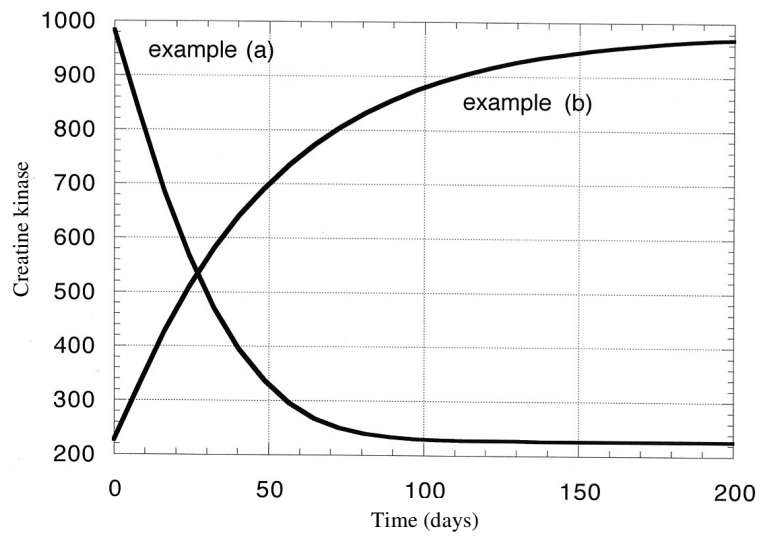

Fig. 5: Example (a): Initial CK-value $=983.33 \mathrm{Ul} / \mathrm{L}$, $\mathrm{P}=250.0 \mathrm{Ul} / \mathrm{L}$, the steroid $(\mathrm{S})=62.5 \mathrm{mg}$ every second day and the natural product is taken daily. Example (b): Initial CK-value $=226.63$, $\mathrm{P}=26.56$ and $\mathrm{S}=0$

26.56 and $\mathrm{S}=0$. The calculated CK-value increases with time to reach a constant value of $\mathrm{CK}=983.33$ $(\mathrm{P}=250.0)$.

Our findings support other studies indicating that up-regulation of CK-MB and cTnT is common in inflammatory muscle diseases ${ }^{[7-10,19]}$. The source of the elevated cTnT has not been established, however there are a number of possibilities. First, although cTnT is not found in healthy adult skeletal muscle ${ }^{[20]}$, it is present in regenerating muscle fibers ${ }^{[21]}$. Second, it is possible that cTnT is re-expressed in skeletal muscle. Finally, one needs to consider the potential crossreactivity between the cardiac and skeletal isoforms of troponin $\mathrm{T}$. However the specificity of the assay towards cTnT has been confirmed ${ }^{[6]}$.

Our findings indicate that the increase in CK-MB was consistent and sustained over a long period of time that is highly indicative of a neuromuscular pathology ${ }^{[22]}$. It has previously been reported that chronic degeneration and regeneration of skeletal muscle leads to an increased release of CK-MB from the skeletal muscle into the bloodstream ${ }^{[22]}$.

Our evidence suggests that we may interpret our results by including the following additional processes in our model, namely:

$$
\begin{array}{ll}
\mathrm{M} \rightarrow \mathrm{Y}+\mathrm{M}+\mathrm{Z} & \left(\mathrm{k}_{\mathrm{a}}\right) \\
\mathrm{Y} \rightarrow \mathrm{Z} & \left(\mathrm{k}_{\mathrm{b}}\right)
\end{array}
$$

where, $\mathrm{Y}$ represents CK-MB and troponin $\mathrm{T}$.

Furthermore, we wish to emphasise that our model addresses the cases when (i) the CK may not be related to the muscle damage, (ii) the CK may be related to the muscle damage, (iii) the steroid interacts with the chemicals attacking the muscles, and (iv) the steroid does not interact with the chemicals attacking the muscles.

In summary, our data reinforce the finding that plasma levels of CK-MB and cTnT cannot be used as an indicator of myocardial disease in patients with skeletal muscle diseases such as polymyositis.

\section{ACKNOWLEDGEMENTS}

We wish to acknowledge the continued support from the Queensland Medical Laboratories for collecting the required blood samples on a six-weekly basis and the corresponding pathology reports.

\section{REFERENCES}

1. Golding, E.M. and R.M. Golding, 2002. Controlling and monitoring polymyositis under steroid therapy. Med. Hypoth., 59: 674-681.

2. Golding, R.M. and L.G.F. Giles, 2006, A method for extracting muscle information from serum creatine kinase measurements-its potential value in the monitoring and management of inflammatory muscle disease. Med. Hypoth., 66: 476-485.

3. Engel, G. and S.G. Rockson, 2007. Rapid diagnosis of myocardial injury with troponin $\mathrm{T}$ and CK-MB relative index. Mol. Diagn. Ther., 11: 109-116.

4. Keshgegian, A.A. and N.V. Feinberg, 1984. Serum creatine kinase MB isoenzyme in chronic muscle disease. Clin. Chem., 30: 575-578.

5. Adams, J.E. 3rd, D.R. Abendschein and A.S. Jaffe, 1993. Biochemical markers of myocardial injury. Is MB creatine kinase the choice for the 1990s? Circulation, 88: 750-763.

6. Muller-Bardorff, M., K. Hallermayer and A. Schroder et al., 1997. Improved troponin T ELISA specific for cardiac troponin $\mathrm{T}$ isoform: Assay development and analytical and clinical validation. Clin. Chem., 43: 458-466.

7. Erlacher, P., A. Lercher, J. Falkensammer et al., 2001. Cardiac troponin and beta-type myosin heavy chain concentrations in patients with polymyositis or dermatomyositis. Clin. Chim. Acta, 306: 27-33.

8. Hamilton, J.S. and P.C. Sharpe, 2005. Two cases of inflammatory muscle disease presenting with raised serum concentrations of troponin T. J. Clin. Pathol., 58: 1323-1324.

9. Lindberg, C., L. Klintberg and A. Oldfors, 2006. Raised troponin $\mathrm{T}$ in inclusion body myositis is common and serum levels are persistent over time. Neuromuscul. Disord., 16: 495-497. 
10. Schwarzmeier, J.D., A. Hamwi and M. Preisel et al., 2005. Positive troponin T without cardiac involvement in inclusion body myositis. Hum. Pathol., 36: 917-921.

11. Khavandi, A., N.P. Jenkins, H.S. Lee and M. Gavalas, 2005. Misdiagnosis of myocardial infarction by troponin I following minor blunt chest trauma. Emerg. Med. J., 22: 603-604.

12. Lavoinne, A. and G. Hue, 1998. Serum cardiac troponins $\mathrm{I}$ and $\mathrm{T}$ in early posttraumatic rhabdomyolysis. Clin. Chem., 44: 667-668.

13. Golding, E.M. and R.M. Golding, 2001. An insight into cortisol and polymyositis control with steroid therapy. Med. Hypoth., 57: 76-86.

14. Golding, R.M., L.G.F. Giles and E.M. Sokoya, 2006. Polymyositis initiation involving amlodipine besylate. Am. J. Immun., 2: 19-22.

15. Golding, R.M., 2003. Polymyositis-a further investigation. Physiol. Meas., 24: 859-878.

16. Barohn, R.J., A.A. Amato, Z. Sahen, J.T. Kissel and J.R. Mendell, 1995. Inclusion body myositis: Explanation for poor response to immunosuppressive therapy. Neurology, 45: 1302-1304.
17. Akatsuka, N., T. Yamaguchi and M. Matsuda, 1974. Long-term effect of corticosteroids on cardiac function. Jpn. Heart, J., 15: 443-454.

18. Golding, E.M. and R.M. Golding, 2001. Mathematical modelling of responses of cerebral blood vessels to changing intraluminal pressure. Physiol. Meas., 22: 727-743.

19. Mahalingam, M. and M.E. Ottlinger, 1996. A case against the specificity of cardiac troponin-T. J. Clin. Pathol., 49: 766-767.

20. Anderson, P.A., N.N. Malouf, A.E. Oakeley, E.D. Pagani and P.D. Allen, 1991, Troponin T isoform expression in humans. A comparison among normal and failing adult heart, fetal heart, and adult and fetal skeletal muscle. Circ. Res., 69: 1226-1233.

21. Bodor, G.S., L. Survant, E.M. Voss, S. Smith, D. Porterfield and F.S. Apple, 1997. Cardiac troponin $\mathrm{T}$ composition in normal and regenerating human skeletal muscle. Clin. Chem., 43: 476-484.

22. Arenas, J., V. Diaz and G. Liras et al., 1988. Activities of creatine kinase and its isoenzymes in serum in various skeletal muscle disorders. Clin. Chem., 34: 2460-2462. 